\title{
e-Phaïstos
}

e-Phaïstos

Revue d'histoire des techniques / Journal of the history

of technology

IV-2 | 2015

Patrimoines de l'eau

\section{L'hydraviation allemande et austro-hongroise dans la Première Guerre mondiale}

German and Austro-Hungarian seaplanes in the first World War

\section{Marion Weckerle}

\section{OpenEdition}

Journals

Édition électronique

URL : http://journals.openedition.org/ephaistos/787

DOI : 10.4000/ephaistos.787

ISSN : 2552-074

Éditeur

IHMC - Institut d'histoire moderne et contemporaine (UMR 8066)

Édition imprimée

Date de publication : 1 octobre 2015

ISSN : 2262-7340

Référence électronique

Marion Weckerle, «L'hydraviation allemande et austro-hongroise dans la Première Guerre mondiale ", e-Phaïstos [En ligne], IV-2 | 2015, mis en ligne le 01 octobre 2015, consulté le 19 avril 2019. URL : http:// journals.openedition.org/ephaistos/787 ; DOI : 10.4000/ephaistos.787

Ce document a été généré automatiquement le 19 avril 2019

Tous droits réservés 


\section{L'hydraviation allemande et austro- hongroise dans la Première Guerre mondiale}

German and Austro-Hungarian seaplanes in the first World War

Marion Weckerle

$1 \mathrm{Si}$, au sein de la discipline de l'histoire des techniques, en particulier dans les problématiques traitant de transmission de savoir-faire et de gestes ${ }^{1}$, les liens entre les différentes pensées techniques ont été étudiés par Anne-Françoise Garçon ${ }^{2}$, Hélène Vérin ${ }^{3}$ ou encore Cyril Lacheze ${ }^{4}$, peu de travaux ont été réalisés sur la période contemporaine. Celle-ci voit pourtant l'émergence de complexes techniques extrêmement élaborés. En particulier, les aéronefs plus lourds que l'air, pilotés par des sportifs dont des compétiteurs accomplis dans d'autres moyens de locomotion - l'automobile ou la bicyclette - provoquent un engouement particulier en Europe pour la discipline aérienne émergente à l'orée $\mathrm{du} \mathrm{XX}^{\mathrm{e}}$ siècle ${ }^{5}$. Les différentes puissances visant à se doter d'une aéronautique performante, particulièrement la France et l'Allemagne, favorisent l'innovation dans ce champ technique par les échanges commerciaux, selon des modalités variées.

2 Le premier chapitre de l'histoire aérienne est indissociable de la Première Guerre mondiale, contexte marquant la lutte industrielle des différents belligérants pour conserver une supériorité technologique conséquente sur les autres nations, aboutissant à une guerre totale. L'intérêt renouvelé, dans le champ académique de l'histoire, pour le travail industriel accompli à l'arrière ${ }^{6}$, fait de ce conflit un terrain historique d'investigation approprié pour interroger les différents savoirs et pensées techniques, à travers les régimes de la pensée opératoire. En suivant Anne-Françoise Garçon, nous en distinguons trois majeurs: le régime de la pratique, fondé sur le savoir-faire transmis oralement, le régime de la technique, formalisant les pratiques par l'écrit, et enfin le régime de la technologie au sein duquel la conception systématisée et mathématisée des produits intervient en amont de leur fabrication ${ }^{7}$. Ces trois axes ont été étudiés 
individuellement dans l'historiographie, mais ils ne sont cependant que très rarement considérés en tant qu'ensembles qui s'interpénètrent.

3 Les ouvrages sur le développement de l'aviation durant la Première Guerre mondiale sont nombreux, et les travaux universitaires concernant l'histoire de l'aéronautique se multiplient avec notamment un groupe dédié à ces questions au sein de l'équipe d'histoire des techniques de l'Université Paris $\mathrm{I}^{8}$. Pourtant, l'analyse de la place de l'hydravion dans ce conflit se fait discrète. La quasi-absence de recherche transversale empêche de saisir certaines dynamiques de la pensée technique spécifique au développement de l'hydro-aéroplane. De même, il ne peut être expliqué le caractère aujourd'hui très spécialisé de l'hydravion, représenté principalement par le Canadair, ainsi que l'engouement pour les hydravions de transport transatlantiques de l'entredeux-guerres jusque dans les années 1950, s'il n'est pas fait état des processus techniques industriels et commerciaux antérieurs.

4 L'aviation, et plus particulièrement l'hydraviation, en tant que carrefour de lignées techniques - ne serait-ce que de l'avion et du bateau - constitue un sujet de premier intérêt pour aborder cette thématique. On voit en effet, de l'immédiat avant-guerre à l'après-guerre, les machines évoluer de modèles uniques pensés et construits de manière empirique, à de véritables productions en série purement technologiques. Nous nous concentrons en particulier sur l'Allemagne et l'Autriche-Hongrie, lesquels constituent un bloc géopolitique cohérent dans ce cadre, mais avec chacune des doctrines différenciées en matière d'hydraviation. Ceci n'empêche pas non plus les transferts techniques ${ }^{9}$ de différentes natures entre elles, l'Allemagne fournissant à l'Autriche-Hongrie des licences de production et du matériel jusqu'au démantèlement de l'empire des Habsbourg.

5 La période d'avant-guerre 1912-1915 apparaît comme une époque formative de l'hydraviation avec les vols et les mises en compétition des premiers appareils en Europe. La discipline se divise d'emblée en des cultures techniques différentes selon le pays considéré, notamment en ce qui concerne la conception de l'appareil. En effet, certains appareils utilisent des flotteurs, quand d'autres ont une coque conçue directement pour supporter un amerrissage. Deux lignées techniques se dessinent ainsi. Par ailleurs, l'utilisation de différents matériaux, ou encore la structure générale de la cellule de l'avion distinguent aussi différentes variantes et dessinent d'autres lignées.

6 Qu'il s'agisse de l'Allemagne ou de l'Autriche-Hongrie, l'origine du développement de l'hydraviation nationale est à rechercher au moins en partie dans un emprunt à un pays extérieur, un transfert technique au sens propre, mais exclusivement à travers l'objet fini. En effet, si quelques firmes allemandes ont commencé à produire des hydravions à flotteurs de leur propre ressort dès le début de l'année $1912^{10}$, en adaptant des flotteurs aux avions terrestres à la place du train d'atterrissage, l'adoption définitive de cette lignée pour les hydravions allemands provient au moins en partie d'un choix officiel au niveau étatique, suite à l'essai réussi d'un appareil britannique à flotteurs. Les constructeurs ont ainsi pour consigne de reproduire ses caractéristiques ${ }^{11}$.

7 À quelques exceptions près, ce choix technique reste valable jusqu'à l'après-guerre ${ }^{12}$. Les hydravions Dornier et Fokker seuls s'essaient à la coque, mais très ponctuellement et sans application opérationnelle ${ }^{13}$. Le même constat est valable pour l'emploi de l'acier et de l'aluminium dans tout ou partie de l'appareil, sérieusement envisagé au moins par Dornier mais encore expérimental ${ }^{14}$. Les autres hydravions disponibles, principalement des Friedrichshafen, améliorent leurs performances d'une génération à l'autre principalement au niveau de la motorisation ${ }^{15}$. 
8 Du côté austro-hongrois, un phénomène similaire a lieu, avec une logique plus simple dans la mesure où cet empire ne dispose que d'un seul constructeur d'importance d'hydravions: Lohner. Celui-ci, s'étant inspiré d'appareils français à coque pour ses premières productions, et ce avec succès, continue à exploiter la même lignée technique, avec là encore des caractéristiques globales communes aux différentes versions ${ }^{16}$.

9 Du point de vue du pilotage et de l'opérationnel, la culture technique des deux pays semble principalement dictée par les impératifs du moment, avec une adéquation parfois assez relative entre les préconisations officielles et la réalité. Ainsi, alors que les appareils étaient censés pouvoir être employés comme avions de reconnaissance dotés de TSF et d'appareils photographiques, ou encore pour des missions de bombardement dès 1913 en Allemagne, rien n'est, dans la pratique, prévu pour cela lorsque la guerre est déclarée ${ }^{17}$. Les dirigeables sont d'ailleurs nettement plus en vue pour la reconnaissance. Les Allemands ne montent un réel groupement opérationnel pour l'hydravion qu'à la fin de l'année 1914. Dans les deux cas, les missions se limitent principalement à la reconnaissance et l'accompagnement de bateaux, avec un développement progressif du bombardement et l'installation de mitrailleuses à bord courant 1915, liées en particulier à l'entrée en guerre de l'Italie dans le cas de l'Autriche-Hongrie ${ }^{18}$. Cependant, les confrontations réelles entre aéronefs restent éloignées de l'image d'Épinal du duel aérien. La grande majorité des pertes d'équipage et de matériels sont imputables à des problèmes mécaniques, de motorisation en particulier. Au combat, la défense anti-aérienne provoque plus de dégâts que la rencontre d'un avion ennemi ${ }^{19}$.

10 Les débuts de l'hydraviation en Allemagne et en Autriche-Hongrie peuvent donc être envisagés comme un cas d'école d'apparition de deux lignées techniques (à flotteurs ou à coque adaptée) via des transferts techniques différents dans la construction des avions. Le rôle, d'une part de l'appareil gouvernemental et administratif, d'autre part d'une firme en situation de quasi-monopole, peuvent également être mis en exergue. La culture technique dans l'emploi des appareils est en revanche plus proche dans les deux pays, quoique non identique, dictée par les impératifs assez semblables sur les deux fronts.

11 L'historiographie de l'aéronautique et de la Grande Guerre considère généralement l'année 1916 comme un tournant décisif. Une guerre, que l'on pensait courte, dure, les fronts se multiplient et prennent de l'ampleur. Cette année voit effectivement l'apparition en opérations d'aviation et d'hydraviation de chasse, ainsi que l'installation d'un nouveau front maritime stratégique dans l'Adriatique. Des programmes aéronautiques plus ambitieux se préparent avec notamment la multiplication des escadrilles et l'intensification de la production en série en usine.

12 Ainsi, on passe de missions de petite envergure destinées à appuyer les opérations navales, en particulier dans la mer du Nord, à des batailles navales nécessitant de rechercher l'innovation technique dans l'arme aérienne afin de surprendre l'ennemi et prendre l'avantage. Ce trait définit la période 1916-1918 comme une nouvelle phase de la guerre, voire un nouveau type de guerre résolument axée sur les percées techniques que les industriels peuvent proposer. Le véritable enjeu ne se situe plus dans la tranchée mais se déplace dans les complexes industriels de l'arrière. Il peut se définir comme suit: les organisations techniques disponibles permettent-elles d'élaborer des productions techniques adéquates aux exigences des différents théâtres opérationnels? Dans quelle mesure les exigences structurelles d'un aéroplane, et a fortiori d'un hydro-aéroplane, peuvent-elles concorder avec les besoins du terrain? Dans ce cadre, la pensée expérimentale, empirique, portée par des praticiens de l'hydraviation, ne suffit plus à 
assurer la production industrielle. La nécessaire rapidité de l'industrialisation de l'hydraviation commande ainsi un changement radical de façon de penser et d'organiser la conception et la production. Cela accélère le passage à un régime technologique de pensée opératoire qui s'observe dans les sources par la différence de ton et la façon différente de poser les problèmes techniques à résoudre.

Dans l'Adriatique, l'hydravion appuie considérablement les opérations navales, en particulier lors de la bataille du détroit d'Otrante ${ }^{20}$. Dans la mer du Nord, on attend plus désormais de l'avion et du pilote que du marin, alors que les flottes britannique et allemande continuent de s'affronter ${ }^{21}$. Par ailleurs, l'hydravion serait un aéronef à l'intérêt amoindri si l'on se contentait d'une comparaison stricte avec les capacités de son pendant terrestre, comme dans le cas des bombardiers, puisque l'hydravion a alors une capacité d'emport de charge utile plus faible. L'hydravion se pose comme un aéroplane très particulier, composite et essentiellement marin ${ }^{22}$. On compte en outre des innovations plus conséquentes comme l'inversion du sens de la gouverne de direction, notamment sur l'hydravion biplan Hansa-Brandenburg W29, permettant au mitrailleur de queue d'avoir à la fois une meilleure visibilité et une meilleure capacité offensive à l'arrière de l'avion ${ }^{23}$.

14 L'expérience acquise les années précédentes permet à des formules techniques d'aéroplane bien connus de se généraliser. En Allemagne, c'est particulièrement celle du biplan, biplace, monocoque, avec le moteur situé à l'avant voire dans le nez et une hélice tractive, qui se révèle la mieux maîtrisée pour répondre aux impératifs de solidité et de performance ${ }^{24}$.

15 Toutefois, les constructeurs ne s'exemptent pas pour autant de modifications inspirées des essais des premiers aéronefs, comme le phénomène de résurgence des triplans en 1916, menant des aéronefs pourtant anecdotiques en terme de nombre de machines utilisées à participer largement au mythe de la figure de l'aviateur, en particulier le Fokker Dr. I côté allemand. Ce caractère technique, une fois transféré à l'aviation maritime avec des prototypes dessinés par Ernst Heinkel ou encore la firme Friedrichshafen, reste pourtant une niche de faible ampleur en regard des dizaines de modèles proposés par les constructeurs allemands, tout comme la discrète lignée expérimentale d'avions à quatre voire cinq plans ${ }^{25}$. D'autres démarches visant à resserrer les liens entre la marine et l'hydraviation, avec un projet d'hydravion embarqué sur sousmarin, ne parviennent pas à concilier les éléments du complexe technique de la mobilité - savoir-faire, matières premières et chaînes de production - avec les exigences posées par le contexte opérationnel visé26.

16 À la signature de l'armistice, des programmes de construction d'avions sont toujours en cours dans les usines de l'arrière. La signature du Traité de Versailles, qui met fin à la guerre, est lourde de conséquences pour l'hydraviation allemande qui doit, de par les restrictions et réparations imposées, trouver de nouvelles voies pour se développer dans un contexte civil cette fois. Quelles sont les modalités de l'hydraviation civile aprèsguerre ? De quelles pensées techniques découle-t-elle?

17 Le contexte géopolitique des années 1919 à 1925 montre un profond remaniement des relations diplomatiques entre les principales puissances européennes ainsi que de nouveaux pouvoirs émergents. Celles-ci modèlent pour une grande part la teneur des relations commerciales entre les différents pays, et donc leurs échanges en matière d'aéronautique dans une période d'expansion des lignes aériennes, employées au transport de courrier ou de personnes. 
18 L'Allemagne, avec les handicaps posés par les conditions de paix qu'elle doit contourner pour s'imposer en tant que puissance aéronautique, en particulier dans le domaine des hydravions en alliages légers métalliques, constitue un cas d'étude particulièrement intéressant pour cerner l'évolution de la pensée technique dans le champ de la locomotion aérienne. Différents éléments du complexe technique migrent - qu'il s'agisse d'appareils vendus ou dissimulés, d'usines ou encore de filiales de sociétés fondées à l'étranger. Des coopérations, contribuant au renouvellement des savoirs techniques avec l'expatriation d'aviateurs et d'ingénieurs, succèdent à la rivalité guerrière, dont le spectre n'est cependant pas totalement absent des préoccupations - conséquence logique de son rôle de premier plan dans la Première Guerre mondiale ${ }^{27}$.

La volonté d'empêcher un réarmement de l'Allemagne, concrétisé par les restrictions et contrôles des commissions interalliées, contraste avec la volonté de ne pas contrecarrer totalement l'avancement des connaissances. Bien que certains la perçoivent comme un danger, les acteurs aéronautiques français sont bel et bien conscients de l'avance prise par l'Allemagne dans le domaine de l'hydraviation de transport, particulièrement grâce à ses procédés de traitement du duralumin ${ }^{28}$.

Plusieurs évolutions de la pensée technique relative à l'aéronautique sont alors palpables dans notre analyse. Premièrement, le passage définitif du régime de la pratique à celui de la technologie est bien observé. Il aurait été intéressant d'observer dans quelle mesure les savoir-faire des ouvriers d'usine ont été influencés, mais le corpus disponible pour l'étude ne permet pas de traiter cette question. Deuxièmement, nous sommes face à deux pensées, deux démarches, qui se traduisent par deux tons différents employés dans les sources françaises, qui se gênent mutuellement. L'Allemagne parvient à pointer les failles du traité et à les exploiter. Des éléments de ses complexes techniques migrent, sous la forme de matériel, de personnes et de leurs savoirs, en valorisant un domaine de recherche spécialisé.

21 Les résultats obtenus par celui-ci ainsi que ses développements ultérieurs, sortant du cadre de la présente étude, marquent une avancée décisive dans l'histoire technique de l'hydravion.

\section{BIBLIOGRAPHIE}

BECKER Jean-Jacques, «L'évolution de l'historiographie de la Première Guerre mondiale », Revue historique des armées, 2006, n²42, p. 4-15.

CHADEAU Emmanuel, L'industrie aéronautique en France, 1900 - 1950. De Blériot à Dassault, Paris, Fayard, 1987, 552 p.

CIGLIĆ Boris, Seaplanes of Bocche, the story of Austro-Hungarin Naval Aviation in the Southern Adriatic 1913 - 1918, Beograd, Jeroplan Books, 2014, 144 p.

CRESSWELL Robert, « Transferts de techniques et chaînes opératoires 》, Techniques et Cultures, 2, 1983, p. $143-163$. 
CULPEPPER David, L'armée de l'air en 1940 : comment et pourquoi ?, mémoire de Master 2 en Histoire des techniques à l'Université Paris I, sous la direction d'Anne-Françoise GARÇON, 2014, $200 \mathrm{p}$.

FORST Günter, KÖSSLER Karl, KOOS Volker, Dornier, von den Anfängen bis 1945, Königswinter, Heel, 2010, 199 p.

GARÇON Anne-Françoise, « Réduire la mine en sciences ? Anatomie des De re metallica (1528 1556) », in DUBOURG-GLATIGNY Pascal, VÉRIN Hélène (éds.), Réduire en Art. La technologie de la Renaissance aux Lumières, Paris, éditions de la MSH, 2008, p. 317-335.

GARÇON Anne-Françoise, L'imaginaire et la pensée technique. Une approche historique, XVIe XXe siècle, Paris, Classiques Garnier, 2012, 286 p.

GRASSER Pierre, Coopérations franco-russes dans l'industrie aéronautique civile, 1903 - 2014, mémoire de Master 2 en Relations internationales à l'Université Paris IV, sous la direction de Pascal GRISET, 2014, 164 p.

GREY Peter, THETFORD Owen, German Airraft of the First World War, Putnam, 1962, 600 p. GROSZ Peter, « A study in contrasts - German submarine aircraft of World War One », Air Enthusiast, $n^{\circ} 33,1987$, p. 1-8.

HALPERN Paul G, The Battle of the Otranto Straits. Controlling the Gateway to the Adriatic in World War I, Indiana University Press, Bloomington, 2004, 186 p.

HERRIS Jack, German Seaplane Fighters of WWI, Aeronaut Books, 2013, 150 p.

IMRIE Alex, German Naval Air Service, London, Weidenfeld Military, 1989, 48 p.

JAKAB Peter, « Wood to Metal: The Structural Origins of the Modern Airplan », Journal of Aircraft, $n^{\circ}$ 6, vol. 36, novembre-décembre 1999, p. 914 - 918.

JANE Fred, Jane's all the World's Aircraft, London, Jane' Information Group, 1913, 436 p.

KOOS Volker, Die Fokker Flugzuegwerke in Schwerin, Geschichte - Produktion - Typen, Schwerin, Thon, 1993, 38 p.

LACHEZE Cyril, L'art du briquetier, XVIe - XIXe siècle. Construction d'une pensée technique à partir d'un savoir-faire. Du régime de la pratique au régime de la technique, thèse de doctorat en cours en Histoire des techniques à l'Université Paris I, sous la direction d'Anne-Françoise GARÇON.

LEAMAN Paul, « The Hansa-Brandenburg seaplanes, part 1 - the early lettered designations », Cross and Cockade International, vol 41, 2010, p $245-259$.

MÜCKLER Jörg, Deutsche Flugzeuge im Ersten Weltkrieg, Stuttgart, Motorbuch Verlag, 2013, $223 \mathrm{p}$.

PAWLAS Karl, Deutsche Flugzeuge, 1914 - 1918, Nürnberg, Luffahrt Dokumente, 1976, 320 p.

PÈLEGRIN Jacques, « Les savoir-faire : une très longue histoire », Terrain, 16, 1991, p. 106-113.

Porte Rémy, La mobilisation industrielle, premier front de la Grande Guerre ? Paris, éditions 14 $18,2006,368 \mathrm{p}$.

TREADWELL Terry, German an austro-hungarin aircraft manufacturers, 1908 - 1918, Amberley, 2011, 256 p.

VÉRIN Hélène, La Gloire des ingénieurs. L'intelligence technique du XVIe au XVIIIe siècle, Paris, Albin Michel, 1993, 456 p. 
WECKERLE Marion, L'hydraviation allemande et austro-hongroise dans la Première Guerre mondiale, mémoire de Master 2 en Histoire des techniques à l'Université Paris I, sous la direction d'Anne-Françoise GARÇON, 2015.

WECKERLE Marion, « Dissimuler le développement dans une entreprise aéronautique », communication orale lors de la masterclasse « Les visites d'entreprises », 11 avril 2015, Université Paris I.

WECKERLE Marion, « Hansa-Brandenburg CC : la chasse sur coque », Air Magazine, à paraître.

\section{Sources}

Service Historique de la Défense (SHD), Direction de l'Armée de Terre, Vincennes :

Série 2012 ZL 245, fonds Émile Dorand,

Commission Interalliée de Contrôle Aéronautique :

Série 7 N, État-Major de l'Armée, 2e bureau.

\section{NOTES}

1. PÈLEGRIN Jacques, « Les savoir-faire : une très longue histoire », Terrain, 16, 1991, p. 106-113.

2. GARÇON Anne-Françoise, «Réduire la mine en sciences? Anatomie des De re metallica (1528 1556), in DUBOURG-GLATIGNY Pascal, VÉRIN Hélène (éds.), Réduire en Art. La technologie de la Renaissance aux Lumières, Paris, éditions de la MSH, 2008, p. 317-335.

3. VÉRIN Hélène, La Gloire des ingénieurs. L'intelligence technique du XVI ${ }^{e}$ au XVIII ${ }^{e}$ siècle, Paris, Albin Michel, 1993, $456 \mathrm{p}$.

4. LACHEZE Cyril, L'art du briquetier, XVI - XIX ${ }^{e}$ siècle. Construction d'une pensée technique à partir d'un savoir-faire. Du régime de la pratique au régime de la technique, thèse de doctorat en cours en Histoire des techniques à l'Université Paris I, sous la direction d'Anne-Françoise GARçON.

5. CHADEAU Emmanuel, L'industrie aéronautique en France, 1900 - 1950. De Blériot à Dassault, Paris, Fayard, 1987, $552 \mathrm{p}$.

JANE Fred, Jane's all the World's Aircraft, London, Jane' Information Group, 1913, 436 p. WECKERLE Marion, L'hydraviation allemande et austro-hongroise dans la Première Guerre mondiale, mémoire de Master 2 en Histoire des techniques à l'Université Paris I, sous la direction d'Anne-Françoise GARÇON, 2015, 209 p.

6. BECKER Jean-Jacques, "L'évolution de l'historiographie de la Première Guerre mondiale ", Revue historique des armées, 2006, $\mathrm{n}^{\circ} 242$, p. 4-15.

Porte Rémy, La mobilisation industrielle, premier front de la Grande Guerre? Paris, éditions 14-18, 2006, $368 \mathrm{p}$.

7. GARÇON Anne-Françoise, L'imaginaire et la pensée technique. Une approche historique, $\mathrm{XVI}^{e}-\mathrm{XX}^{e}$ siècle, Paris, Classiques Garnier, 2012, 286 p.

8. CULPEPPER David, L'armée de l'air en 1940: comment et pourquoi ?, mémoire de Master 2 en Histoire des techniques à l'Université Paris I, sous la direction d'Anne-Françoise GARÇON, 2014, 200 p. GRASSER Pierre, Coopérations franco-russes dans l'industrie aéronautique civile, 1903 - 2014, mémoire de Master 2 en Relations internationales à l'Université Paris IV, sous la direction de Pascal GRISET, 2014, 164 p. WECKERLE Marion, L'hydraviation allemande et austro-hongroise dans la Première Guerre mondiale, mémoire de Master 2 en Histoire des techniques à l'Université Paris I, sous la direction d'Anne-Françoise GARÇON, 2015, 209 p.

9. CRESSWELL Robert, " Transferts de techniques et chaînes opératoires ", Techniques et Cultures, 2, 1983, p. $143-163$. 
10. JANE Fred, Jane's all the World's Aircraft, London, Jane' Information Group, 1913, $436 \mathrm{p}$.

11. MÜCKLER Jörg, Deutsche Flugzeuge im Ersten Weltkrieg, Stuttgart, Motorbuch Verlag, 2013, 223 p. WECKERLE Marion, L'hydraviation allemande et austro-hongroise dans la Première Guerre mondiale, mémoire de Master 2 en Histoire des techniques à l'Université Paris I, sous la direction d'Anne-Françoise GARÇON, 2015, 209 p.

12. JANE Fred, Jane's all the World's Aircraft, London, Jane' Information Group, 1913, 436 p. MÜCKLER Jörg, Deutsche Flugzeuge im Ersten Weltkrieg, Stuttgart, Motorbuch Verlag, 2013, 223 p. PAWLAS Karl, Deutsche Flugzeuge, 1914 - 1918, Nürnberg, Luffahrt Dokumente, Publizistiches Archiv Karl Pawlas, 1976, 320 p. TREADWELL Terry, German an austro-hungarin aircraft manufacturers, 1908 - 1918, Amberley, 2011, 256 p. WECKERLE Marion, L'hydraviation allemande et austro-hongroise dans la Première Guerre mondiale, mémoire de Master 2 en Histoire des techniques à l'Université Paris I, sous la direction d'Anne-Françoise GARÇON, 2015, 209 p.

13. KOOS Volker, Die Fokker Flugzuegwerke in Schwerin, Geschichte - Produktion - Typen, Schwerin, Thon, 1993, $38 \mathrm{p}$.

JAKAB Peter, "Wood to Metal: The Structural Origins of the Modern Airplan », Journal of Aircraft, $\mathrm{n}^{\circ}$ 6, vol. 36, novembre-décembre 1999, p. 914 - 918.

14. FORST Günter, KÖSSLER Karl, KOOS Volker, Dornier, von den Anfängen bis 1945, Königswinter, Heel, 2010, 199 p.

15. Lieutenant d'artilleries GILLES et al, Rapport technique de la Commission de Contrôle Aéronautique en Allemagne, vol 1, titre 2, Hydravions, s.d [1920], 102 p. SHD, Série DE 2012 ZL 245, fonds Émile Dorand, carton $n^{\circ} 19$.

16. CIGLIĆ Boris, Seaplanes of Bocche, the story of Austro-Hungarin Naval Aviation in the Southern Adriatic 1913 - 1918, Beograd, Jeroplan Books, 2014, 144 p.

17. JANE Fred, Jane's all the World's Aircraft, London, Jane' Information Group, 1913, 436 p.

18. IMRIE Alex, German Naval Air Service, London, Weidenfeld Military, 1989, 48 p. WECKERLE Marion, L'hydraviation allemande et austro-hongroise dans la Première Guerre mondiale, mémoire de Master 2 en Histoire des techniques à l'Université Paris I, sous la direction d'Anne-Françoise GARÇON, 2015, 209 p.

19. CIGLIĆ Boris, Seaplanes of Bocche, the story of Austro-Hungarin Naval Aviation in the Southern Adriatic 1913 - 1918, Beograd, Jeroplan Books, 2014, 144 p.

20. HALPERN Paul G, The Battle of the Otranto Straits. Controlling the Gateway to the Adriatic in World War I, Indiana University Press, Bloomington, 2004, 186 p.

WECKERLE Marion, « Hansa-Brandenburg CC : la chasse sur coque », Air Magazine, à paraître.

21. HERRIS Jack, German Seaplane Fighters of WWI, Aeronaut Books, 2013, 150 p. LEAMAN Paul, The Hansa-Brandenburg seaplanes, part 1 - the early lettered designations, Cross and Cockade International, vol 41, 2010, p 245 - 259.

22. MÜCKLER Jörg, Deutsche Flugzeuge im Ersten Weltkrieg, Stuttgart, Motorbuch Verlag, 2013, 223 p. WECKERLE Marion, L'hydraviation allemande et austro-hongroise dans la Première Guerre mondiale, mémoire de Master 2 en Histoire des techniques à l'Université Paris I, sous la direction d'Anne-Françoise GARÇON, 2015, 209 p.

23. HERRIS Jack, German Seaplane Fighters of WWI, Aeronaut Books, 2013, 150 p.

24. WECKERLE Marion, L'hydraviation allemande et austro-hongroise dans la Première Guerre mondiale, mémoire de Master 2 en Histoire des techniques à l'Université Paris I, sous la direction d'AnneFrançoise GARÇON, 2015, 209 p.

25. GREY Peter, THETFORD Owen, German Airraft of the First World War, Putnam, 1962, 600 p.

PAWLAS Karl R, Deutsche Flugzeuge, 1914-1918, Nürnberg, Luffahrt Dokumente, 1976, 320 p.

26. GROSZ Peter, "A study in contrasts - German submarine aircraft of World War One ", Air Enthusiast, n³3, 1987, p. 1-8.

27. WECKERLE Marion, "Dissimuler le développement dans une entreprise aéronautique ", communication orale lors de la masterclasse "Les visites d'entreprises », 11 avril 2015, 
Université Paris I. WECKERLE Marion, L'hydraviation allemande et austro-hongroise dans la Première Guerre mondiale, mémoire de Master 2 en Histoire des techniques à l'Université Paris I, sous la direction d'Anne-Françoise GARÇON, 2015, 209 p.

28. SHD, Série DE 2012 ZL 245, fonds Émile Dorand, Vincennes.

\section{RÉSUMÉS}

Notre article présente de manière synthétique les résultats de la recherche conduite lors de notre Master 2 au sein de l'équipe d'Histoire des techniques de l'Université Paris I. Nous analysons l'émergence, dans les principales puissances européennes et belligérantes de la Première Guerre mondiale, et le rôle de cet aéronef composite combinant des caractéristiques techniques et opérationnelles à la fois de l'avion et du navire. En nous concentrant sur l'Allemagne impériale et l'Autriche-Hongrie, nous avons pu mettre en lumière différentes lignées techniques et doctrines. L'Allemagne tire avantage du nombre de ses constructeurs aéronautiques, multiplie les modèles et compte considérablement sur ses hydravions de chasse en mer du Nord. Tandis que l'AutricheHongrie, dans l'Adriatique, se repose sur quelques modèles d'hydravions à coque qu'elle est quasiment la seule puissance à employer.

Nous pouvons distinguer trois périodes chronologiques parallèlement au déroulement de la Première Guerre mondiale. Les premiers hydravions allemands et autrichiens documentées apparaissent en 1912, basés sur des modèles étrangers. Jusqu'en 1915, les travaux sont essentiellement expérimentaux et les hydravions ne connaissent pas encore de réelle application guerrière, malgré des spécifications demandées par les États-Majors. La période 1916 - 1918 représente un tournant avec l'Italie qui a choisi son camp, ainsi que la production d'aéroplanes se chiffrant à des dizaines puis des centaines de machines par mois. Une formule générale et techniquement maîtrisée d'aéroplane est privilégiée par les empires centraux. Les performances des hydravions s'avèrent néanmoins inférieures à celles des avions terrestres.

À la signature du Traité de Versailles, l'Allemagne doit cesser toute son activité aéronautique, mais met au point des stratégies pour contourner les failles du traité. Tandis que des machines sont livrées aux puissances alliées ou détruites, d'autres sont cachées ou développées à l'étranger. Les ingénieurs migrent donc avec leur savoir et leur savoir-faire, en particulier dans le domaine des grands hydravions de transport métalliques. En 1925, l'Allemagne, ayant tiré parti du nouveau contexte géopolitique européen, a retrouvé une place importante dans le marché civil aéronautique et travaille sur des hydravions transatlantiques.

This paper introduces the results of the research conducted during our Master's degree at University Paris I, in the history of techniques team. We analyze the emergence of the seaplane in the main European powers and the involvement of this composite craft combining technical and operational specifications of aircraft and ships. Focusing on Imperial Germany and its ally the Austro-Hungarian empire, we were thus able to investigate the different paths taken: Germany, with many manufacturers, relies on fighter floatplanes in the North Sea, whereas Austria and Hungary, in the Adriatic sea, count mostly on a few types of flying boats, of which the empire is practically the unique user.

We can distinguish three chronological periods, following the outbreak and the evolution of World War One. The first documented known seaplanes in Germany and Austria appear in 1912, based on foreign machines. Until 1915, the work is highly experimental and the seaplanes have 
no real martial application, despite of the specifications required. 1916 - 1918 represents a major turn with the choice of Italy and the production in series of dozens, and soon hundreds, aircrafts per month. A general and technically mastered formula of plane is privileged. Still, the performances of the seaplanes, comparatively to the landplanes, could seem uninteresting.

At the signature of the Versailles Treaty, Germany has to cease activity in aeronautics, especially for warring purposes, but still finds strategies to carry on work in this field. Whereas aircraft are delivered to Allies or destroyed, others are hidden or developed abroad. Engineers migrate with their specialized knowledge in the large, metallic transport seaplanes. By 1925, Germany has found an important place in civil aviation in the new geopolitical European context and works on transatlantic seaplanes.

INDEX

Mots-clés : histoire des techniques, 20e siècle, Europe, hydravion, régime de pensée opératoire, guerre

Keywords : history of technology, 20th century, Europa, sea-plane, technology thinking scheme, war

\section{AUTEUR}

\section{MARION WECKERLE}

Doctorante - Équipe d'Histoire des Techniques / IHMC/CNRS-Université Paris 1 PanthéonSorbonne 